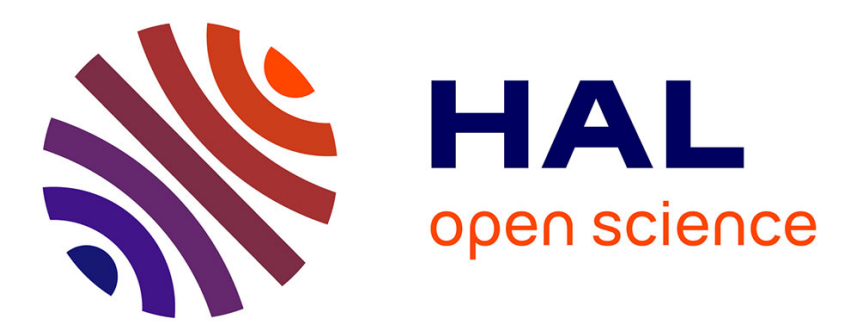

\title{
A Stability Index for Local Effectivity Functions
}

Joseph M. Abdou

\section{To cite this version:}

Joseph M. Abdou. A Stability Index for Local Effectivity Functions. 2008. halshs-00331223

\section{HAL Id: halshs-00331223 \\ https://shs.hal.science/halshs-00331223}

Submitted on 15 Oct 2008

HAL is a multi-disciplinary open access archive for the deposit and dissemination of scientific research documents, whether they are published or not. The documents may come from teaching and research institutions in France or abroad, or from public or private research centers.
L'archive ouverte pluridisciplinaire HAL, est destinée au dépôt et à la diffusion de documents scientifiques de niveau recherche, publiés ou non, émanant des établissements d'enseignement et de recherche français ou étrangers, des laboratoires publics ou privés. 


\section{Documents de Travail du Centre d'Economie de la Sorbonne}

C

E

$\mathrm{S}$

W

0

1

k

$\frac{i}{g}$

$\mathrm{P}$

a

$\mathrm{p}$

e

$r$

$\mathrm{S}$

CENTRE NATIONAL

DE LA RECHERCHE

SCIENTIFIQUE 


\title{
A Stability Index for Local Effectivity Functions
}

\author{
Joseph Abdou *
}

July 15, 2008

\begin{abstract}
We study the structure of unstable local effectivity functions defined for $n$ players and $p$ alternatives. A stability index based on the notion of cycle is introduced. In the particular case of simple games, the stability index is closely related to the Nakamura Number. In general it may be any integer between 2 and $p$. We prove that the stability index for maximal effectivity functions and for maximal local effectivity functions is either 2 or 3 .
\end{abstract}

Keywords: Stability Index, Acyclicity, Strong Nash Equilibrium, Core, Solvability, Consistency, Simple Game, Effectivity Function.

JEL Classification: C70, D71 AMS Classification: 91A44

*Ecole d'Economie de Paris, Paris 1, CNRS, 106-112 boulevard de l'Hôpital 75647 Paris Cedex 13 - France; email: abdou@univ-paris1.fr 


\section{Introduction}

Stability is a highly desirable property for political systems. The modeling of political interactions has to take this stability requirement into account. In coalitional models, stability is defined as the possibility of achieving, for any preference profile, a state that no coalition would oppose. In strategic models, this amounts to the existence, for any preference profile, of an equilibrium (solvability). However it is commonly known that most political systems are unstable in this sense. In mathematical social sciences, results known as impossibility theorems reflect the fact that stability (or solvability) is rather hard to obtain. Therefore it is interesting to investigate the properties of unstable mechanisms.

Although this investigation can be carried out in a far more general setting (see Abdou and Keiding (2003) for the general notion of effectivity structure), we shall focus in this study on the so-called local effectivity functions. While effectivity functions appear naturally in the study of implementation theory (Moulin and Peleg, 1982; Peleg and Winter, 2002; Peleg, 2004; Peleg and Peters, 2008), as well as in the theory of Constitutions and Rights (Peleg, 1998), local effectivity functions are closely related to the solvability problem (Abdou, 1995, 2000). In their investigation of game form solvability (e.g. for Nash or strong Nash equilibrium), Abdou and Keiding (2003) pointed out two new aspects regarding the power distribution: (1) the dependence of this power on the current state (the local aspect), (2) the joint character of the interactive power (the interactive aspect). In this paper we limit ourselves to the local aspect only, but instead of deriving our object from a strategic game form, we define it abstractly. Like an effectivity function, a local effectivity function describes the power of coalitions to achieve an outcome in some subsets of alternatives, but unlike an effectivity function, this power may depend on the current state. Our choice to restrict our attention on this object is justified by its simplicity, its natural interpretation and the elegance of the properties involved in its analysis. However and despite this restriction, the local aspect adds an original ingredient and reveals new properties that cannot be articulated if we limit the study to effectivity functions. Stability of local effectivity functions is defined as the non-vacuity of the core for all preference profiles.

Necessary and sufficient conditions for stability of local effectivity functions may be deduced as a particular case of stability of effectivity structures (Abdou and Keiding, 2003, Theorem 6) and therefore, they are not the central question of this article. Here our main objective is to further investigate, what can be said when the local effectivity function is unstable. The notion of cycle lies at the heart of stability characterization. This fact, known since Condorcet, turns out to be very general. A cycle (definition 3.1) is a sequence of potential objections that obeys a combinatorial property that guarantees their compatibility. The existence of a cycle is equivalent to the 
existence of some profile for which any potential state is opposed by some coalition. Therefore, to study instability we must explore the structure of cycles.

As a first step in our investigation of instability, we introduce a stability index defined precisely as the mimimal length of all possible cycles $(+\infty$ in case of stability). This number does not exhaust all the features of instability of a local effectivity function, but does provide a meaningful classification of instability types. If the cardinal of the alternative set is $p$, the stability index can be any integer between 2 and $p$. In this paper we identify the index of some subclasses of local effectivity functions. In the case of simple games, our index can be viewed as the analog of the Nakamura number (Nakamura, 1979). Indeed, when the stability index is finite then it coincides with the Nakamura number (Corollary 4.5). The second class for which we determine the stability index is that of maximal effectivity functions (Theorem 4.7). This is done by checking classical properties: regularity, superadditivity and subadditivity. Our method for the general case consists in extracting two appropriate effectivity functions from the local effectivity function, and to check whether they coincide. When this is the case, the local effectivity function is said to be exact. Again, in the class of maximal local effectivity functions, we can determine the stability index: by checking exactness and classical properties of effectivity functions (Theorem 4.13). It is remarkable that for maximal effectivity functions and indeed for maximal local effectivity functions, the stability index is either 2 or 3 .

The paper is organized as follows: In Section 2, local effectivity functions and related concepts are defined. Cycles are the main object of Section 3. Section 4 is devoted to the study of the stability index. Its relationship to the Nakamura number is established in Subsection 4.1. The determination of the stability index of maximal effectivity functions is the object of Subsection 4.2 and that of maximal local effectivity functions is the object of Subsection 4.3. We conclude in Section 5.

\section{The model}

In this section we define a model of interaction that specifies the power distribution of a set of agents over some set of alternatives, with no explicit reference to any strategic mechanism that gives rise to that power. We shall see later (definition 2.5) how, starting from a strategic mechanism (i.e. a game form) one can derive an appropriate description of the power distribution induced by the strategies. The notions that we present in this section, have in common that only the independent power held by coalitions is represented. They are encompassed by the concept of local effectivity function. We shall see that the latter includes, as particular cases, effectivity functions and simple games. 


\subsection{Basic notations}

Throughout this paper we shall consider a finite set $N$, the elements of which are called players or agents and a finite set $A$, the elements of which are called alternatives or states. We make use of the following notational conventions: For any set $D$, we denote by $\mathcal{P}(D)$ the set of all subsets of $D$ and by $\mathcal{P}_{0}(D)=\mathcal{P}(D) \backslash\{\emptyset\}$ the set of all non-empty subsets of $D$. Elements of $\mathcal{P}_{0}(N)$ are called coalitions. $N \backslash S$ is denoted $S^{c}$. Similarly if $B \in \mathcal{P}(A)$, $A \backslash B$ is denoted $B^{c} . L(A)$ will denote the set of all linear orders on $A$ (that is all binary relations on $A$ which are complete, transitive, and antisymmetric). If $R \in L(A)$, and $a, b \in A, a \neq b, a R b$ means that $a$ is better than $b$ in the linear order $R$. A preference profile (over $A$ ) is a map from $N$ to $L(A)$, so that a preference profile is an element of $L(A)^{N}$. For every preference profile $R_{N} \in L(A)^{N}$ and $S \in \mathcal{P}_{0}(N)$ we put

$$
P\left(a, S, R_{N}\right)=\left\{b \in A \mid b \neq a, b R^{i} a, \forall i \in S\right\}
$$

(so that $P\left(a, S, R_{N}\right)$ consists of all the outcomes considered to be better than $a$ by all members of the coalition $S)$, and $P^{c}\left(a, S, R_{N}\right)=A \backslash P\left(a, S, R_{N}\right)$.

\subsection{Local effectivity functions}

In the study of game form solvability, the idea that the power of a coalition may depend on the current state, arises naturally. This is the reason why a local effectivity function was first introduced in Abdou (1995), but only as an object related to a game form and an equilibrium concept (e.g. Nash or strong Nash). In this paper we shall work with an abstract coalitional form, where the power of coalitions depends on the current state.

Definition 2.1 A local effectivity function on $(N, A)$ is a family $\mathcal{E} \equiv(\mathcal{E}[U]$, $\left.U \in P_{0}(A)\right)$ where for any $U \in P_{0}(A), \mathcal{E}[U]: \mathcal{P}(N) \rightarrow \mathcal{P}\left(\mathcal{P}_{0}(A)\right)$ and such that the following conditions are satisfied:

(i) $\mathcal{E}[U](S)=\emptyset$ if and only if $S=\emptyset$,

(ii) $B \in \mathcal{E}[U](S), B \subset B^{\prime} \Rightarrow B^{\prime} \in \mathcal{E}[U](S)$

(iii) $U \subset V \Rightarrow \mathcal{E}[V](S) \subset \mathcal{E}[U](S)$

The formula $B \in \mathcal{E}[U](S)$ is interpreted as follows: When the current state is in $U$, coalition $S$ can adapt its response in order to realize some state in $B$. Let $R_{N} \in L(A)^{N}$. An alternative $a \in A$ is dominated at $R_{N}$ if there exists $U \in \mathcal{P}_{0}(A), S \in \mathcal{P}_{0}(N)$ such that $a \in U$ and $P\left(a, S, R_{N}\right) \in \mathcal{E}[U](S)$. The core of $\mathcal{E}$ at $R_{N}$ is the set of undominated alternatives. It is denoted $C\left(\mathcal{E}, R_{N}\right) . \mathcal{E}$ is stable if $C\left(\mathcal{E}, R_{N}\right) \neq \emptyset$ for all $R_{N} \in L(A)^{N}$. We introduce a partial order on the set of all local efectivity functions on $(N, A)$ as follows: $\mathcal{E} \preceq \mathcal{E}^{\prime}$ if and only if : $\forall U \in \mathcal{P}_{0}(A), \forall S \in \mathcal{P}_{0}(N): \mathcal{E}[U](S) \subset \mathcal{E}^{\prime}[U](S)$. 
Local effectivity functions that appear in the litterature satisfy some natural properties: The first one is monotonicity w.r.t. players:

(m) $\forall U \in \mathcal{P}_{0}(A), \forall S \in \mathcal{P}_{0}(N), \forall T \in \mathcal{P}_{0}(N): S \subset T \Rightarrow \mathcal{E}[U](S) \subset \mathcal{E}[U](T)$

The second, Possibility of Non-Action, is defined as follows:

(a) $\forall U \in \mathcal{P}_{0}(A), \forall S \in \mathcal{P}_{0}(N): U \in \mathcal{E}[U](S)$

The third one is the sheaf property:

$(s) \forall S \in \mathcal{P}_{0}(N), \forall U \in \mathcal{P}_{0}(A): \mathcal{E}[U](S)=\cap_{a \in U} \mathcal{E}[\{a\}](S)$

Although they may play a role in some circumstances, these properties are not needed for the most part of this study. In the following remark we show their impact on the core correspondence:

Remark 2.2 Let $(x)$ be any of the properties $(m),(a),(s)$. Given any local effectivity function $\mathcal{E}$ we denote by $\mathcal{E}^{(x)}$ the smallest (for $\preceq$ ) local effectivity function $\mathcal{E}^{\prime}$ that satisfies property $(x)$ and such that $\mathcal{E} \preceq \mathcal{E}^{\prime}$. If we note $\mathcal{E}^{(x y)}$ the result on $\mathcal{E}$ of the operation $(x)$ followed by the operation $(y)$, it is easy to see that that $\mathcal{E}^{(x x)}=\mathcal{E}^{(x)}$ and $\mathcal{E}^{(x y)}=\mathcal{E}^{(y x)}(x, y \in\{m, a, s\})$. Moreover for any $R_{N} \in L(A)^{N}$ and any $x \in\{m, a, s\}$ one has: $C\left(\mathcal{E}^{(x)}, R_{N}\right)=C\left(\mathcal{E}, R_{N}\right)$. One can prove that given two local effectivity functions $\mathcal{E}$ and $\mathcal{F}, C\left(\mathcal{E}, R_{N}\right)=$ $C\left(\mathcal{F}, R_{N}\right)$ for all $R_{N} \in L(A)^{N}$ if and only if $\mathcal{E}^{(\text {mas })}=\mathcal{F}^{(\text {mas })}$.

We now present some notions that appear in social choice theory and show how they can be viewed as particular cases of local effectivity functions. The following can be traced back to Moulin and Peleg (1982):

Definition 2.3 An effectivity function on $(N, A)$ is a mapping $E: \mathcal{P}(N) \rightarrow$ $\mathcal{P}\left(\mathcal{P}_{0}(A)\right.$ such that :

(i) $E(S)=\emptyset$ if and only if $S=\emptyset$,

(ii) $B \in E(S), B \subset B^{\prime} \Rightarrow B^{\prime} \in E(S)$

To any effectivity function $E$, we shall associate the local effectivity function defined by: $\mathcal{E}[U]=E$ for any $U \in \mathcal{P}_{0}(A)$. In an effectivity function the power of a coalitions is independent of the current state. An effectivity function is the analog of a cooperative game with abstract payoffs.

Our second notion is a generalization of simple games as defined in Nakamura (1975):

Definition 2.4 A local simple game on $(N, A)$ is a collection $\mathcal{W}=\left(W_{a}, a \in\right.$ $A)$ where $W_{a} \subset \mathcal{P}_{0}(N),(a \in A)$. When $W_{a}=W$ for all $a \in A$, we have a (standard) simple game $(W, A)$

$W_{a}$ is the set of winning coalitions at $a$. The interpretation of a local simple game is as follows: if the current state is $a$ then any coalition in $W_{a}$ have 
the power to react so that to reach any $b \in A$. For $U \in \mathcal{P}_{0}(A)$, put $\mathcal{W}[U]:=$ $\cap_{a \in U} W_{a}$. To any local simple game, we associate a local effectivity function that reflects the same power distribution. It is defined by $\mathcal{E}[U](S)=\mathcal{P}_{0}(A)$ if $S \in \mathcal{W}[U]$ and $\mathcal{E}[U](S)=\{A\}$ if $S \notin \mathcal{W}[U], S \neq \emptyset$. In a local simple game, given some current state, a coalition is either totally powerful or totally powerless.

The third notion comes from strategic game theory. We consider a strategic game form $G=\left\langle\left(X_{i}\right)_{i \in N}, A, g\right\rangle$ where $X_{i}$ is the strategy set of player $i$, $(i \in N)$ and $g: \prod_{i \in N} X_{i} \rightarrow A$ is the outcome function. We assume that $g$ is onto. If $S \in \mathcal{P}_{0}(N)$ we denote by $X_{S}$ the cartesian product $\prod_{i \in S} X_{i}$. If $x_{N} \in X_{N}$ we also write $x_{N}=\left(x_{S}, s_{S^{c}}\right)$.

Definition 2.5 Let $G$ be a strategic game form. The local effectivity function $\mathcal{E}^{G}$ associated to $G$ is defined as follows: For $U \in \mathcal{P}_{0}(A), \mathcal{E}^{G}[U](\emptyset)=\emptyset$ and for $S \in \mathcal{P}_{0}(N)$ :

$$
\mathcal{E}^{G}[U](S)=\left\{B \in \mathcal{P}_{0}(A) \mid \forall x_{N} \in g^{-1}(U), \exists y_{S} \in X_{S}: g\left(x_{S^{c}}, y_{S}\right) \in B\right\}
$$

The $\beta$-effectivity function associated to $G$ is defined by the formula: $E_{\beta}^{G}=$ $\mathcal{E}^{G}[A]$.

The local effectivity function associated to a strategic game form was first introduced in Abdou (2000) in relation to strong Nash solvability. It satisfies properties $(m),(a)$ and $(s)$.

\section{3 cycles}

The main tool used in the analysis of stability is the notion of cycle. In the case of effectivity functions, it has been defined in Keiding (1985). Later on this notion has been extended to more general structures (Abdou and Keiding 2003).

Definition 3.1 An $r$-tuple $\left(\left(C_{1}, B_{1}, S_{1}\right), \ldots,\left(C_{r}, B_{r}, S_{r}\right)\right)$ where $r \geq 1$, $C_{k} \in \mathcal{P}_{0}(A), B_{k} \in \mathcal{P}_{0}(A), S_{k} \in \mathcal{P}_{0}(N)(k=1, \ldots, r)$ is a cycle in $\mathcal{E}$ if :

(i) $B_{k} \in \mathcal{E}\left[C_{k}\right]\left(S_{k}\right)(k=1, \ldots, r)$,

(ii) $\cup_{k=1}^{r} C_{k}=A$,

(iii) For any $\emptyset \neq J \subset\{1, \ldots, r\}$ such that $\cap_{k \in J} S_{k} \neq \emptyset$, there exists $k \in J$ such that for all $l \in J: C_{k} \cap B_{l}=\emptyset$.

$\left(C_{1}, \ldots, C_{r}\right)$ is said to be the basis of the cycle and $r$ its length. If $\left(C_{1}, \ldots, C_{r}\right)$ is a partition of $A$ the cycle is said to be strict. $\mathcal{E}$ is said to be acyclic if it has no cycle. 
Condition (i) states that at any alternative in $C_{k}$, coalition $S_{k}$ can achieve $B_{k}$, and therefore that coalition $S_{k}$ would object against $C_{k}$ if it were in the interest of its members to do so. Condition (iii) is a combinatorial property that insures that the sequence of potential objections is somehow consistent. Condition (ii) is clear. Using the notations of definition 3.1, some immediate consequences are in order:

1) For any cycle: $C_{k} \cap B_{k}=\emptyset(k=1, \ldots, r)$,

2) If $S_{k} \cap S_{l}=\emptyset, k \neq l,(k, l=1, \cdots, r)$ then condition (iii) amounts to $C_{k} \cap B_{k}=\emptyset(k=1, \cdots, r)$,

3) Any cycle has length $\geq 2$,

4) For any cycle: $\cap_{k=1}^{r} S_{k}=\emptyset$,

5) Any cycle of length $r$ gives rise to a strict cycle with length $\leq r$. This can be done as follows: Put $\tilde{C}_{1}=C_{1}$ and $\tilde{C}_{k}=C_{k} \backslash \cup_{l=1}^{k-1} C_{l}(2 \leq k \leq r)$, and remove the indices $k$ corresponding to empty $\tilde{C}_{k}$, and as a consequence:

6) Any cycle has length $\leq|A|$.

Example 3.2 (a) Let $E$ be an effectivity function. An an $r$ - tuple $\left(\left(B_{1}, S_{1}\right)\right.$, $\left.\ldots,\left(B_{r}, S_{r}\right)\right)$ where $r \geq 2, S_{k} \in \mathcal{P}_{0}(N), B_{k} \in E\left(S_{k}\right)(k=1, \ldots, r), B_{k} \cap B_{l}=$ $\emptyset(k \neq l)$ and $\cap_{k=1}^{r} S_{k}=\emptyset$ gives rise to a cycle of $E$. We have a basis by putting: $C_{k}=B_{k-1}, k=2, \ldots, r$ and $C_{1}=A \backslash \cup_{k=2}^{r} C_{k}$.

(b) Let $E$ be an effectivity function. An an $r$-tuple $\left(\left(B_{1}, S_{1}\right), \ldots,\left(B_{r}, S_{r}\right)\right)$ where $r \geq 2, S_{k} \in \mathcal{P}_{0}(N), B_{k} \in E\left(S_{k}\right)(k=1, \ldots, r), S_{k} \cap S_{l}=\emptyset(k \neq l)$ and $\cap_{k=1}^{r} B_{k}=\emptyset$ gives rise to a cycle of $E$. By putting $C_{k}=B_{k}^{c}(k=1, \ldots, r)$ (and removing those indices $\mathrm{k}$ with $B_{k}^{c}=\emptyset$ ) we have a basis.

In the rest of this section we shall express the properties defining a cycle in a few equivalent ways. In what follows, as far as we are concerned with the combinatorial property ( $i i i$ ) of definition 3.1, one can point out a partial duality between the roles of the $B_{k}$ and the $C_{k}$.

Proposition 3.3 In definition 3.1 one can replace condition (iii) by either one of the following conditions:

(iiia) For any $\emptyset \neq J \subset\{1, \ldots, r\}$ such that $\cap_{k \in J} S_{k} \neq \emptyset$, there exists $k \in J$ such that for all $l \in J: B_{k} \cap C_{l}=\emptyset$.

(iiib) For any $\emptyset \neq J \subset\{1, \ldots, r\}$ such that $\cap_{k \in J} S_{k} \neq \emptyset$, there exists a sequence $k_{1}, \cdots, k_{|J|}$ such that $J=\left\{k_{1}, \cdots, k_{|J|}\right\}$, and $\left[\left(C_{k_{1}} \cup \cdots \cup C_{k_{j}}\right)\right] \cap$ $\left[B_{k_{j}} \cup \cdots \cup B_{k_{|J|} \mid}\right]=\emptyset(j=1, \cdots,|J|)$.

Proof. Clearly (iiib) implies both (iii) and (iiia) : by taking $k=k_{1}$ for (iii) and $k=k_{|J|}$ for (iiia). Assume that (iii) is satisfied. Let $\emptyset \neq J \subset\{1, \ldots, r\}$ such that $\cap_{k \in J} S_{k} \neq \emptyset$. There exists $k_{1} \in J$ such that $C_{k_{1}} \cap\left(\cup_{l \in J} B_{l}\right)=\emptyset$. 
If $J \backslash\left\{k_{1}\right\}$ is empty our construction is over. If not let $k_{2} \in J \backslash\left\{k_{1}\right\}$ such that $C_{k_{2}} \cap\left(\cup_{l \in J \backslash\left\{k_{1}\right\}} B_{l}\right)=\emptyset$. By repeating this argument, we construct: $k_{1}, \cdots, k_{|J|}$ such that (iiib) is satisfied. By a dual construction starting from (iiia) it can be proved that (iiib) is satisfied.

A selection of $\mathcal{P}_{0}(\{1, \ldots, r\})$ is a map $\theta: \mathcal{P}_{0}(\{1, \ldots, r\}) \rightarrow\{1, \ldots, r\}$ such that $\theta(J) \in J$, for all $J \in \mathcal{P}_{0}(\{1, \ldots, r\})$. Let $\Sigma_{r}$ be the set of all selections of $\mathcal{P}_{0}(\{1, \ldots, r\})$. To any $\left(S_{1}, \ldots, S_{r} ; A_{1} \ldots A_{r}\right) \in \mathcal{P}_{0}(N)^{r} \times \mathcal{P}_{0}(A)^{r}$, $\theta \in \Sigma_{r}$ and $k \in\{1, \ldots, r\}$ we associate:

$$
\begin{gathered}
\mathcal{J}_{k}^{\theta} \equiv \mathcal{J}_{k}^{\theta}\left(S_{1}, \ldots, S_{r}\right):=\left\{J \in \mathcal{P}_{0}(\{1, \ldots, r\}) \mid \cap_{j \in J} S_{j} \neq \emptyset, \theta(J)=k\right\} \\
\mathcal{A}_{k}^{\theta}\left(S_{1}, \ldots, S_{r} ; A_{1} \ldots A_{r}\right):=A \backslash \bigcup_{J \in \mathcal{J}_{k}^{\theta}} \bigcup_{j \in J} A_{j}
\end{gathered}
$$

In what follows we write $\mathcal{C}_{k}^{\theta}$ for $\mathcal{A}_{k}^{\theta}\left(S_{1}, \ldots, S_{r} ; B_{1} \ldots B_{r}\right)$ and we write $\mathcal{B}_{k}^{\theta}$ for $\mathcal{A}_{k}^{\theta}\left(S_{1}, \ldots, S_{r} ; C_{1} \ldots C_{r}\right)$.

Proposition 3.4 In definition 3.1 condition (iii) can be replaced by:

(iiic) There exists $\theta \in \Sigma_{r}$ such that $C_{k} \subset C_{k}^{\theta}(k=1 \cdots, r)$.

Proof. Straightforward.

If $E$ is an effectivity function, this proposition admits a more elegant formulation:

Proposition 3.5 Let $E$ be an effectivity function and let $\left(S_{1}, \cdots S_{r} ; B_{1}, \cdots\right.$, $\left.B_{r}\right) \in \mathcal{P}_{0}(N)^{r} \times \mathcal{P}_{0}(A)^{r}$ such that $B_{k} \in E\left(S_{k}\right)(k=1, \cdots, r)$. Then the following are equivalent:

(i) There exists $\left(C_{1}, \cdots, C_{r}\right) \in \mathcal{P}_{0}(A)^{r}$ such that $\left(\left(C_{1}, B_{1}, S_{1}\right), \cdots,\left(C_{r}, B_{r}\right.\right.$, $\left.S_{r}\right)$ ) is a cycle of $E$.

(ii) There exists $\theta \in \Sigma_{r}$ such that $\left(\mathcal{C}_{1}^{\theta}, \cdots, \mathcal{C}_{r}^{\theta}\right)$ is a covering of $A$ and $\mathcal{C}_{k}^{\theta} \neq \emptyset$ $(k=1, \cdots, r)$.

Proof. Easy consequence of the preceding proposition and the remark that for an effectivity function, the condition $B_{k} \in E\left(S_{k}\right)$ replaces the condition $B_{k} \in \mathcal{E}\left[C_{k}\right]\left(S_{k}\right)$.

Taking into account the partial duality between the $B_{k}$ and the $C_{k}$ we have the following:

Proposition 3.6 Let $\mathcal{E}$ be a local effectivity function, let $\left(C_{1}, \cdots, C_{r}\right) \in$ $\mathcal{P}_{0}(A)^{r}$ be a covering of $A$ and let $\left(S_{1}, \cdots, S_{r}\right) \in \mathcal{P}_{0}(N)^{r}$. The following are equivalent :

(i) There exists $\left(B_{1}, \cdots, B_{r}\right)$ such that $\left(\left(C_{1}, B_{1}, S_{1}\right), \cdots,\left(C_{r}, B_{r}, S_{r}\right)\right)$ is a cycle of $\mathcal{E}$.

(ii) There exists $\theta \in \Sigma_{r}$ such that $\mathcal{B}_{k}^{\theta} \in \mathcal{E}\left[C_{k}\right]\left(S_{k}\right)(k=1, \cdots, r)$. 
Proof. (i) $\Rightarrow$ (ii). Assume that for some $\left(B_{1}, \cdots, B_{r}\right),\left(\left(C_{1}, B_{1}, S_{1}\right), \cdots,\left(C_{r}\right.\right.$, $\left.\left.B_{r}, S_{r}\right)\right)$ is a cycle. We define $\theta \in \Sigma_{r}$ as follows: By property (iiia) of Proposition 3.3, we put $\theta(J)=k$ if $\cap_{j \in J} S_{j} \neq \emptyset$, and $\theta(J) \in J$ arbitrarily if $\cap_{j \in J} S_{j}=\emptyset$. It follows that for all $J \in \mathcal{J}_{k}^{\theta}, B_{k} \cap\left(\cup_{j \in J} C_{j}\right)=\emptyset$ or equivalently: $B_{k} \subset \mathcal{B}_{k}^{\theta}$. Therefore $\mathcal{B}_{k}^{\theta} \in \mathcal{E}\left[C_{k}\right]\left(S_{k}\right]$.

(ii) $\Rightarrow$ (i). Let $\theta \in \Sigma_{r}$ be such that (ii) is verified. Then it is easy to see that $\left(\left(C_{1}, \mathcal{B}_{1}^{\theta}, S_{1}\right), \ldots,\left(C_{r}, \mathcal{B}_{r}^{\theta}, S_{r}\right)\right)$ is a cycle. In order to check condition (iiia) of Proposition 3.3, let $J$ be such that $\cap_{j \in J} S_{j} \neq \emptyset$ and let $k:=\theta(J)$ then $J \in \mathcal{J}_{k}^{\theta}$ so that $\mathcal{B}_{k}^{\theta} \cap\left(\cup_{i \in J} C_{j}\right)=\emptyset$.

It was asserted earlier (remark 2.2) that if $\mathcal{E}$ is any local effectivity function and if $(x)$ is any of the properties $(m),(a),(s)$ then $\mathcal{E}^{(x)}$ and $\mathcal{E}$ have the same core correspondence. As regards cycles, we have the following :

\section{Remark 3.7}

1) $\mathcal{E}^{(a)}$ and $\mathcal{E}$ have the same cycles,

2) To any cycle of $\mathcal{E}^{(m)}$ corresponds some cycle of $\mathcal{E}$ with the same basis (hence the same length), and vice versa,

3) To any cycle of $\mathcal{E}$ corresponds some cycle of $\mathcal{E}^{(s)}$ (not necessarily with the same length), and vice versa.

We end this section by stating the main result that justifies the introduction of cycles. In the case of effectivity functions, it was first proved by Keiding (1985) (see also Abdou and Keiding, 1991, Theorem 5.3). For a more general result that covers the case of local effectivity functions we refer to Abdou and Keiding (2003), Theorem 6.

Theorem 3.8 A local effectivity function $\mathcal{E}$ is stable if and only if it is acyclic.

\section{$4 \quad$ Instability and the stability index}

This section is devoted to the study of unstable local effectivity functions. It would be interesting to have a typology of the essential characteristics of instability. As a first step toward this clarification, we provide an index that sheds light on the structure of situations that generate instability. Although cycles of the same length may be very different in structure (see example 3.2 where cycle (b) seems to be "simpler" than cycle (a)) we put forward, as a first idea in our investigation, that this index has to do with the length of cycles that may appear in the power distribution.

Definition 4.1 The stability index of $\mathcal{E}$, denoted $\sigma(\mathcal{E})$, is the minimal length of a cycle in $\mathcal{E} . \sigma(\mathcal{E})$ is set to $+\infty$ if $\mathcal{E}$ is acyclic. 
Let $f: A \rightarrow A^{\prime}$ be a map. Let $\mathcal{E}$ be a local effectivity function on $(N, A)$. We define the image $\mathcal{E}^{f}$ of $\mathcal{E}$ by $f$ as the local effectivity function on $\left(N, A^{\prime}\right)$ where, for any $U^{\prime} \in \mathcal{P}_{0}\left(A^{\prime}\right)$ :

$$
\mathcal{E}^{f}\left[U^{\prime}\right](S)=\left\{B^{\prime} \in \mathcal{P}_{0}\left(A^{\prime}\right) \mid f^{-1}\left(B^{\prime}\right) \in \mathcal{E}\left[\left(f^{-1}\left(U^{\prime}\right)\right](S)\right\}\right.
$$

The $r$-tuple $\left(\left(C_{1}^{\prime}, B_{1}^{\prime}, S_{1}\right), \ldots,\left(C_{r}^{\prime}, B_{r}^{\prime}, S_{r}\right)\right)$ is a cycle of $\mathcal{E}^{f}$ if and only if $\left(\left(f^{-1}\left(C_{1}^{\prime}\right), f^{-1}\left(B_{1}^{\prime}\right), S_{1}\right), \ldots,\left(f^{-1}\left(C_{r}^{\prime}\right), f^{-1}\left(B_{r}^{\prime}\right), S_{r}\right)\right)$ is a cycle of $\mathcal{E}$. It follows that $\mathcal{E}^{f}$ is acyclic if $\mathcal{E}$ is acyclic.

Conversely, let $\left(\left(C_{1}, B_{1}, S_{1}\right), \ldots,\left(C_{r}, B_{r}, S_{r}\right)\right)$ be a cycle of $\mathcal{E}$ based on the partition $\left(C_{1}, \ldots, C_{r}\right)$. Let $A^{\prime}$ be some set with $r$ elements $A^{\prime}:=\left\{u_{1}, \ldots, u_{r}\right\}$ and let $f: A \rightarrow A^{\prime}$ be defined by $f(a)=u_{k}$ if $a \in U_{k}$. Put $B_{k}^{\prime}:=f\left(B_{k}\right)$ $k=1, \cdots, r$. For any $k, l \in\{1, \ldots, r\}$ one has $U_{k} \cap B_{l}=\emptyset$ if and only if $\left\{u_{k}\right\} \cap f\left(B_{l}\right)=\emptyset$. It follows that $\left(\left\{\left(u_{1}\right\}, B_{1}^{\prime}, S_{1}\right), \ldots,\left(\left\{u_{r}\right\}, B_{r}^{\prime}, S_{r}\right)\right)$ is a cycle of $\mathcal{E}^{f}$ based on the partition $\left(\left\{u_{1}\right\}, \ldots,\left\{u_{r}\right\}\right)$. Therefore we have the following characterization:

Theorem 4.2 The stability index of a local effectivity function $\mathcal{E}$ is the smallest integer $s$ for which the following property holds:

There exists a surjection $f: A \rightarrow\{1, \cdots, s\}$ such that $\mathcal{E}^{f}$ is unstable.

Proof. Let $s$ be the number defined in the claim. Then for some $f: A \rightarrow$ $\{1, \cdots, s\}, \mathcal{E}^{f}$ has a cycle. By the first part of the above argument $\mathcal{E}$ has a cycle of the same length so that : $\sigma(\mathcal{E}) \leq s$. By the second part of the above argument, since there exists a cycle of length $\sigma(\mathcal{E})$ in $\mathcal{E}$, there exists a surjection $f: A \rightarrow\{1, \cdots, \sigma(\mathcal{E})\}$ such that $\mathcal{E}^{f}$ is unstable, so that $s \leq \sigma(\mathcal{E})$. It follows that $s=\sigma(\mathcal{E})$.

This characterization allows for an interpretation of the stability index. Assume that a local effectivity function is unstable with a stability index $\sigma$, then merging some alternatives results in a transformation of the local effectivity function in a way that respects the power distribution. This is the interpretation of the operation $\mathcal{E} \rightarrow \mathcal{E}^{f}$. This transformation may occur, for instance, when the agents cease to distinguish between two previously distinct alternatives. If the cardinal of the new set is inferior to $\sigma$, then the new local effectivity function is stable. If $\sigma=2$, then instability takes a particularly simple form: alternatives can be partitioned into two aggregates, or two major issues, on which the society is split, and the power of coalitions allowed by the rules is such that both issues can be opposed and neither one can be forced. In general cycles produced by a split of the society into disjoint coalitions, are rather simple. However when $\sigma$ is high, some configurations leading to instability may be combinatorially complex and in order to produce them, the society must have rather sophisticated views. The question of whether the probability (for instance when the preferences 
are assumed to be uniformly distributed) of reaching a cyclic configuration is related to the stability index remains open and is not addressed in this paper. However this relationship, if ever it exists, is not straightforward as can be seen from what follows:

Remark 4.3 In view of remark $3.7, \mathcal{E}^{(a)}, \mathcal{E}^{(m)}$ and $\mathcal{E}$ have the same stability index, but not necessarily $\mathcal{E}^{(s)}$. If follows that two local effectivity functions (e.g. $\mathcal{E}$ and $\mathcal{E}^{(s)}$ ) may have the same core correspondence but different stability indices. Indeed when $\mathcal{E} \neq \mathcal{E}^{(s)}$ some preference profile may generate a cycle in $\mathcal{E}^{(s)}$ that is shorter than any one it generates in $\mathcal{E}$.

\subsection{Stability Index and the Nakamura Number}

In the case of simple games there is a relationship between the stability index and the Nakamura number as defined in Nakamura (1979). This relationship casts light on the stability index and shows that, in a sense, the stability index may be viewed as the analog of the Nakamura number for local effectivity functions. Let $W$ be some set of winning coalitions. $\left(S_{1}, \ldots, S_{r}\right)$ where $S_{k} \in W(k=1 \cdots, r)$ is a said to be a non intersecting family of $W$ if $\cap S_{k=1}^{r}=\emptyset$. The Nakamura Number of $W$, denoted $\nu(W)$, is defined as the minimum length of a non intersecting family. If $W$ has no non intersecting family, then we set $\nu(W)=+\infty$. Let $\mathcal{W}$ be a local simple game on $(N, A)$ as in definition 2.4. A $2 r$ - tuple $\left(U_{1}, S_{1}, \ldots, U_{r}, S_{r}\right)$ where $U_{k} \in \mathcal{P}_{0}(A), S_{k} \in \mathcal{W}\left[U_{k}\right](k=1, \ldots, r)$ is said to be a cycle of $\mathcal{W}$ if $\left(U_{1}, \cdots, U_{r}\right)$ is a partition of $A$ and $\cap S_{k=1}^{r}=\emptyset$. The natural number $r$ is the length of the cycle. We recall that the local effectivity function associated to $\mathcal{W}$ (resp. $(W, A))$ is $\mathcal{E}^{\mathcal{W}}\left(\operatorname{resp} . \mathcal{E}^{W, A}\right)$. Let $\sigma(\mathcal{W})(\operatorname{resp} . \sigma(W, A))$ denote the stability index $\sigma\left(\mathcal{E}^{\mathcal{W}}\right)$ (resp. $\sigma\left(\mathcal{E}^{W, A}\right)$ ). One has the following:

Lemma 4.4 Any cycle of $\mathcal{W}$ gives rise to some strict cycle of $\mathcal{E}^{\mathcal{W}}$ of the same length and vice versa.

Proof. Let $\left(U_{1}, S_{1}, \ldots, U_{r}, S_{r}\right)$ be a cycle in $\mathcal{W}$. Indices are taken in $\mathbb{Z} / r \mathbb{Z}$. Let $B_{k}:=U_{k+1}(k \in \mathbb{Z} / r \mathbb{Z})$. We claim that $\left(\left(U_{1}, B_{1}, S_{1}\right), \ldots,\left(U_{r}, B_{r}, S_{r}\right)\right)$ is a strict cycle of $\mathcal{E}^{\mathcal{W}}$ : In order to prove condition (iii) of definition 3.1 we remark that if $J$ is such that $\cap_{j \in J} S_{j} \neq \emptyset$ then $J \neq\{1, \cdots, r\}$ and we can choose any $k \in J$ such that $k+1 \notin J$. Conversely any strict cycle $\left(U_{1}, B_{1}, S_{1}, \ldots, U_{r}, B_{r}, S_{r}\right)$ in $\mathcal{E}^{\mathcal{W}}$ is such that $\left(U_{1}, \cdots, U_{r}\right)$ is a partition of $A$ and $\cap S_{k=1}^{r}=\emptyset$.

Corollary 4.5 For any simple game $(W, A)$ one has :

$$
\begin{aligned}
\sigma(W, A) & =\nu(W) \quad \text { if } \quad \nu(W) \leq|A| \\
& =+\infty \quad \text { if } \quad \nu(W)>|A|
\end{aligned}
$$

In particular $(W, A)$ is stable if and only if $\nu(W)>|A|$. 
Proof. Let $\nu:=\nu(W)$ and $\sigma:=\sigma(W, A)$. If $\nu \leq|A|$, let $\left(S_{1}, \cdots, S_{\nu}\right)$ be a non intersecting family of $W$. Let $U_{1}, \cdots, U_{\nu}$ be any partition of $A$, then $\left(U_{1}, S_{1}, \ldots, U_{\nu}, S_{\nu}\right)$ is a cycle of $(W, A)$. It follows that $\sigma \leq \nu$. Conversely any cycle $\left(U_{1}, S_{1}, \ldots, U_{\sigma}, S_{\sigma}\right)$ implies that $\left(S_{1}, \ldots, S_{\sigma}\right)$ is an intersecting family, so that $\nu \leq \sigma$. If $\nu>|A|$ then there can be no cycle in $(W, A)$ since there is no partition of $A$ of length $\nu$.

An alternative way to express the relationship between $\sigma(W, A)$ and $\nu(W)$ is as follows:

$$
\begin{aligned}
& \nu(W)=\sigma(W, A) \quad \text { if } \sigma(W, A) \quad<+\infty \\
& >|A| \text { if } \sigma(W, A)=+\infty
\end{aligned}
$$

\subsection{Stability index of Effectivity functions}

It is possible to refine our knowledge of the stability index for some classes of effectivity functions. For that purpose we recall some properties that appear in the study of stability. As will be seen in this subsection they have a fundamental role in the determination of the stability index. An effectivity function $E$ is said to be:

monotonic w.r.t. players if for all $S, T \in \mathcal{P}_{0}(N)$,

$$
S \subset T \Rightarrow E(S) \subset E(T),
$$

regular if for all $S_{1} \in \mathcal{P}_{0}(N), S_{2} \in \mathcal{P}_{0}(N)$,

$$
S_{1} \cap S_{2}=\emptyset, B_{1} \in E\left(S_{1}\right), B_{2} \in E\left(S_{2}\right) \Rightarrow B_{1} \cap B_{2} \neq \emptyset,
$$

maximal if for all $S \in \mathcal{P}_{0}(N), B \in \mathcal{P}_{0}(A)$,

$$
B^{c} \notin E\left(S^{c}\right) \Longrightarrow B \in E(S),
$$

superadditive if for all $S_{1} \in \mathcal{P}_{0}(N), S_{2} \in \mathcal{P}_{0}(N)$,

$$
S_{1} \cap S_{2}=\emptyset, B_{1} \in E\left(S_{1}\right), B_{2} \in E\left(S_{2}\right) \Rightarrow B_{1} \cap B_{2} \in E\left(S_{1} \cup S_{2}\right),
$$

subadditive if for all $S_{1} \in \mathcal{P}_{0}(N), S_{2} \in \mathcal{P}_{0}(N)$,

$$
B_{1} \cap B_{2}=\emptyset, B_{1} \in E\left(S_{1}\right), B_{2} \in E\left(S_{2}\right) \Rightarrow B_{1} \cup B_{2} \in E\left(S_{1} \cap S_{2}\right) .
$$

The $\beta$-Effectivity function $E_{\beta}^{G}$ associated to a strategic game form $G$ (definition 2.5) satisfies monotonicity w.r.t. players and maximality. If an effectivity function is subadditive then it has no cycle of type (a) of example 3.2; it it is superadditive, then it has no cycle ot type (b). It is interesting to note that in the case of maximal effectivity functions, the absence of such cycles (types (a) or (b)) is equivalent to stability. The following clear cut result that can be deduced from Abdou (1982) and Peleg (1984) (Theorem 6.A.9) is reproduced here for future use: 
Theorem 4.6 Let $E$ be a maximal effectivity function. The following are equivalent:

(i) $E$ is stable,

(ii) $E$ is superadditive and subadditive,

(iii) E has no cycles of type (a) or (b) of example 3.2.

As regards the stability index, we can establish, relying on the Theorem 4.6, the following:

Theorem 4.7 Let $E$ be an effectivity function. Then:

(i) $\sigma(E)>2$ if and only if $E$ is regular.

(ii) Assume that $E$ is maximal. Then $\sigma(E) \in\{2,3,+\infty\}$.

Proof. (i) Let $\left(\left(C_{1}, B_{1}, S_{1}\right),\left(C_{2}, B_{2}, S_{2}\right)\right)$ be a 2-cycle. Then $S_{1} \cap S_{2}=$ $\emptyset, C_{1} \cup C_{2}=A, B_{i} \in E\left(S_{i}\right), B_{i} \subset C_{i}^{c}(i=1,2)$, so that $B_{1} \cap B_{2} \subset$ $C_{1}^{c} \cap C_{2}^{c}=\emptyset$. This contradicts regularity. Conversely if $\left(\left(S_{1}, B_{1}\right),\left(S_{2}, B_{2}\right)\right)$ is such that $B_{i} \in E\left(S_{i}\right)(i=1,2), S_{1} \cap S_{2}=\emptyset$ and $B_{1} \cap B_{2}=\emptyset$ then $\left(\left(B_{1}^{c}, B_{1}, S_{1}\right),\left(B_{2}^{c}, B_{2}, S_{2}\right)\right)$ is 2-cycle.

(ii) In view of Theorem 4.6, a maximal effectivity function is stable if and only if it is subadditive and superadditive. In that case $\sigma(E)=+\infty$. If $E$ is not superadditive then there exists $S_{1}, S_{2} \in \mathcal{P}_{0}(N), B_{1}, B_{2} \in \mathcal{P}_{0}(A)$ such that $S_{1} \cap S_{2}=\emptyset, B_{1} \in E\left(S_{1}\right), B_{2} \in E\left(S_{2}\right)$ and $B_{1} \cap B_{2} \notin E\left(S_{1} \cup S_{2}\right)$. Put $S_{3}=\left(S_{1} \cup S_{2}\right)^{c}, B_{3}=\left(B_{1} \cap B_{2}\right)^{c}$. By maximality $B_{3} \in E\left(S_{3}\right)$ so that $\left(\left(B_{1}^{c}, B_{1}, S_{1}\right),\left(B_{2}^{c}, B_{2}, S_{2}\right),\left(B_{3}^{c}, B_{3}, S_{3}\right)\right)$ is a cycle. A similar proof can be done if $E$ is not subadditive. Therefore $\sigma(E) \leq 3$.

\subsection{Stability Index of Local Effectivity Functions}

In order to obtain some precise indications on the stability index of a local effectivity function, we shall define some simpler objects that it induces and that play a role in its stability. In his study of strong Nash solvability, together with the local effectivity functions associated to a game form, Abdou (1995) introduced a property called exactness. The latter generalizes "exact maximality, a property found in $\mathrm{Li}$ (1991). Here we extend this definition to general local effectivity functions. Starting from a local effectivity function $\mathcal{E}$ we define two simpler objets:

The global effectivity function derived from $\mathcal{E}$ is the mapping $E_{0}: \mathcal{P}(N) \rightarrow$ $\mathcal{P}\left(\mathcal{P}_{0}(A)\right)$ such that all $S \in \mathcal{P}(N): E_{0}(S):=\mathcal{E}[A](S)$.

The exact effectivity function derived from $\mathcal{E}$ is the mapping $E_{\xi}: \mathcal{P}(N) \rightarrow$ $\mathcal{P}\left(\mathcal{P}_{0}(A)\right)$ such that $E_{\xi}(\emptyset)=\emptyset$ and for $S \in \mathcal{P}_{0}(S)$, by:

$$
E_{\xi}(S):=\left\{B \in \mathcal{P}_{0}(A) \mid B=A \text { or } \exists a \notin B, B \in \mathcal{E}[\{a\}](S)\right\}
$$


Note that properties (i) and (ii) of definition 2.3 are satisfied by $E_{0}$, whereas $E_{\xi}$ does not necessarily satisfy property (ii) (monotonicity w.r.t. alternatives) so that it is not an "effectivity function". In general it is clear from the definitions that for all $S \in \mathcal{P}_{0}(N), E_{0}(S) \subset E_{\xi}(S)$.

Definition 4.8 A local effectivity function $\mathcal{E}$ is said to be exact if $E_{0}=E_{\xi}$. A local effectivity function $\mathcal{E}$ is said to be maximal if $E_{0}$ is maximal.

Note that the exactness property conveys some information only in the framework of local effectivity function. In the case of effectivity functions, that is, in the case where $\mathcal{E}$ is independent of $U$, it is always true that $E_{0}=E_{\xi}=\mathcal{E}[U]$ (for any $U \in \mathcal{P}_{0}(N)$ ). Exactness together with maximality will shed light on the structure of local effectivity functions with respect to the stability/instability problem. In what follows we investigate the impact of exactness on the core correspondence and cycles of $\mathcal{E}$. Clearly $C\left(\mathcal{E}, R_{N}\right) \subset C\left(E_{0}, R_{N}\right)$ for all $R_{N} \in L(A)^{N}$ and any cycle of $E_{0}$ is a cycle of $\mathcal{E}$. In addition on has:

Proposition 4.9 If $\mathcal{E}$ is exact, then:

(i) $C\left(E_{0}, R_{N}\right)=C\left(\mathcal{E}, R_{N}\right)$ for all $R_{N} \in L(A)^{N}$,

(ii) $\mathcal{E}$ and $E_{0}$ have the same cycles.

Proof. If $a$ is dominated in $\mathcal{E}$ at $R_{N}$, then for some $S \in \mathcal{P}_{0}(N), P\left(a, S, R_{N}\right) \in$ $\mathcal{E}[\{a\}](S)$. Since $a \notin P\left(a, S, R_{N}\right)$, one has $P\left(a, S, R_{N}\right) \in E_{\xi}(S)=E_{0}(S)$ so that $a$ is dominated in $E_{0}$. Likewise if the $r$-tuple $\left(\left(C_{1} B_{1}, S_{1}\right), \ldots,\left(C_{r}, B_{r}, S_{r}\right)\right)$ is a cycle in $\mathcal{E}$, then in particular $C_{k} \cap B_{k}=\emptyset, C_{k} \neq \emptyset$ and since $B_{k} \in$ $\mathcal{E}\left[C_{k}\right]\left(S_{k}\right)$ it follows that $B_{k} \in E_{\xi}\left(S_{k}\right)=E_{0}\left(S_{k}\right)$, so that this $r$-tuple is also a cycle in $E_{0}$.

Lemma 4.10 Assume that $\mathcal{E}$ is maximal and not exact. Then:

(i) there exists $R_{N} \in L(A)^{N}$ such that $C\left(\mathcal{E}, R_{N}\right)=\emptyset$ and if further $E_{0}$ is monotonic w.r.t. players we have in addition $\left|C\left(E_{0}, R_{N}\right)\right|=1$.

(ii) there exists a cycle of length at most 3 in $\mathcal{E}$, that is not a cycle in $E_{0}$.

Proof. (i) Let $S \in P_{0}(N), B \in P_{0}(N)$ such that $B \in E_{\xi}(S)$ and $B \notin E_{0}(S)$. Then there exists $a \in B^{c}$ such that $B \in \mathcal{E}[\{a\}](S)$ and $B^{c} \in E_{0}\left(S^{c}\right)$. Define a profile $R_{N}$ such that: for $i \in S, B R^{i}\{a\} R^{i} B^{c} \backslash\{a\}$ and for $i \in S^{c}$, $\{a\} R^{i} B^{c} \backslash\{a\} R^{i} B$. If $b \in B$ then $P\left(b, S^{c}, R_{N}\right) \supset B^{c} \in E_{0}\left(S^{c}\right)$, so that $b$ is dominated in $E_{0}$. If $b \in B \backslash\{a\}$, then $P\left(b, N, R_{N}\right) \supset\{a\}$. By maximality of $E_{0}, E_{0}(N)=\mathcal{P}_{0}(A)$, so that $b$ is dominated in $E_{0}$. Therefore one has $C\left(E_{0}, R_{N}\right) \subset\{a\}$. Now $P\left(a, S, R_{N}\right)=B \in \mathcal{E}[\{a\}](S)$ implies that $a$ is dominated in $\mathcal{E}$, and since $C\left(\mathcal{E}, R_{N}\right) \subset C\left(E_{0}, R_{N}\right)$ we have $C\left(\mathcal{E}, R_{N}\right)=\emptyset$. If moreover $E_{0}$ is monotonic w.r.t. players, then for $T \in \mathcal{P}_{0}(N), T \subset S$ we 
have $P\left(a, T, R_{N}\right)=B \notin E_{0}(T)$ and for $T \cap S^{c} \neq \emptyset$ we have $P\left(a, T, R_{N}\right)=\emptyset$ so that $a$ is not dominated in $E_{0}$. We conclude that $C\left(E_{0}, R_{N}\right)=\{a\}$.

(ii) Put $S_{1}=S, S_{2}=S^{c}, S_{3}=N, B_{1}=B, B_{2}=B^{c}, B_{3}=\{a\}, C_{1}=$ $\{a\}, C_{2}=B, C_{3}=B^{c} \backslash\{a\}$. In $\mathcal{E}$ this defines a 3-cycle if $C_{3} \neq \emptyset$, and a 2-cycle if $C_{3}=\emptyset$, that is not a cycle in $E_{0}$.

As an immediate consequence of Lemma 4.10 we have the following proposition that provides, when $E_{0}$ is maximal, a partial converse to Proposition 4.9:

Proposition 4.11 Assume that $\mathcal{E}$ is maximal.

(i) If $E_{0}$ is monotonic w.r.t. players then $\mathcal{E}$ is exact if and only if $C\left(E_{0}, R_{N}\right)=$ $C\left(\mathcal{E}, R_{N}\right)$ for all $R_{N} \in L(A)^{N}$.

(ii) $\mathcal{E}$ is exact if and only if $E_{0}$ and $\mathcal{E}$ have the same cycles.

In what follows, we summarize the main results concerning stability of local effectivity functions. This can be viewed as a generalization to the local effectivity functions, of Theorem 3.11 of Abdou (2000) where only objects derived from a game form were considered:

Theorem 4.12 Assume that $\mathcal{E}$ is maximal. Then the following are equivalent:

(i) $\mathcal{E}$ is stable

(ii) $\mathcal{E}$ is exact and $E_{0}$ is stable.

(iii) $\mathcal{E}$ is exact and $E_{0}$ is superadditive and subadditive.

Moreover in that case $C\left(E_{0}, R_{N}\right)=C\left(\mathcal{E}, R_{N}\right)$ for all $R_{N} \in L(A)^{N}$.

Proof. If $\mathcal{E}$ is stable then $E_{0}$ is stable and by the first part of assertion (i) of Lemma $4.10, \mathcal{E}$ is exact. Conversely if $\mathcal{E}$ is exact, then $C\left(E_{0}, R_{N}\right)=$ $C\left(\mathcal{E}, R_{N}\right)$ for all $R_{N} \in L(A)^{N}$ by Lemma 4.9 assertion (i), and if moreover $E_{0}$ is stable it follows that $\mathcal{E}$ is stable. This estabilshes the equivalence between $(i)$ and (ii). The equivalence with (iii) follows from Theorem 4.6.

As regards instability one can obtain precise indications on the index. For that purpose we assert the following:

Theorem 4.13 (i) $\sigma(\mathcal{E})=2$ if $E_{0}$ is not regular.

(ii) Assume that $\mathcal{E}$ is maximal. Then $\sigma(\mathcal{E}) \in\{2,3,+\infty\}$

Proof. If $E_{0}$ is not regular then $2 \leq \sigma(\mathcal{E}) \leq \sigma\left(E_{0}\right)=2$, we conclude that $\sigma(\mathcal{E})=2$. Assume that $E_{0}$ is maximal and $\mathcal{E}$ not stable. Two cases are possible. In the first case $E_{0}$ is stable, then by Theorem $4.12 \mathcal{E}$ is not 
exact and by Lemma 4.10 assertion (ii) $\mathcal{E}$ has a cycle of length $\leq 3$, so that $\sigma(\mathcal{E}) \leq 3$. In the second case $E_{0}$ is not stable then by Theorem $4.7 \sigma\left(E_{0}\right) \leq 3$ and since $\sigma(\mathcal{E}) \leq \sigma\left(E_{0}\right)$ we conclude again that $\sigma(\mathcal{E}) \leq 3$.

Remark 4.14 If $\mathcal{E}$ satisfies the non-action property $(a)$ and the sheaf property $(s)$ (see subsection 2.2 and remark 4.3) then (i) in 4.13 can be improved to read as follows: $\sigma(\mathcal{E})=2$ if and only if $E_{0}$ is not regular: If $\left(\left(C_{1}, B_{1}, S_{1}\right),\left(C_{2}, B_{2}, S_{2}\right)\right)$ is a 2-cycle, then $C_{1} \cup C_{2}=A, S_{1} \cap S_{2}=\emptyset$, $C_{1} \subset B_{1}^{c}$ and $C_{2} \subset B_{2}^{c}, B_{1} \in \mathcal{E}\left[C_{1}\right]\left(S_{1}\right), B_{2} \in \mathcal{E}\left[C_{2}\right]\left(S_{2}\right)$. It follows that $C_{1}^{c} \in \mathcal{E}\left[C_{1}\right]\left(S_{1}\right)$ and $C_{2}^{c} \in \mathcal{E}\left[C_{2}\right]\left(S_{2}\right)$ and in view of properties $(a)$ and $(s)$, $C_{1}^{c} \in \mathcal{E}[A]\left(S_{1}\right)$ and $C_{2}^{c} \in \mathcal{E}[A]\left(S_{2}\right)$. Since $\mathcal{E}[A]=E_{0}, S_{1} \cap S_{2}=\emptyset$ and $C_{1}^{c} \cap C_{2}^{c}=\emptyset$ we deduce that $E_{0}$ is not regular.

\section{Conclusion}

We defined a stability index for local effectivity functions and showed its connection to the Nakamura Number of simple games. We proved that for any unstable maximal local effectivity function that induces a the index is either 2 or 3 . Our results constitute a first step in the comprehension of the nature of instability. It would be interesting to compute the stability index of neutral and anonymous effectivity functions: this is a challenging combinatorial exercice. The same definition of stability index may be extended to more general interaction forms, especially to those derived from strategic game forms. The study of their stability index would give an insight into the nature of instability involved in the underlying equilibrium concept.

\section{References}

Abdou, J., 1982. Stabilité de la fonction veto, Cas du veto maximal. Mathématiques et Sciences Humaines 80, 39 - 63.

Abdou, J., 1995. Nash and strongly consistent two-player game forms. International Journal of Game Theory 24, 345 - 356.

Abdou, J., 2000. Exact stability and its applications to strong solvability. Mathematical Social Sciences 39, 263 - 275.

Abdou, J. and Keiding H., 1991. Effectivity functions in social choice. Kluwer Academic Publishers, Dordrecht.

Abdou, J. and Keiding H., 2003. On necessary and sufficient conditions for solvability of game forms. Mathematical Social Sciences 46, $243-260$.

Keiding, H., 1985. Necessary and sufficient conditions for stability of effectivity functions. International Journal of Game Theory 14, 93 - 101. 
Li, S., 1991. Strongly consistent game forms. Social Choice and Welfare 11, $177-192$.

Moulin, H. and Peleg, B., 1982. Cores of effectivity functions and implementation theory. Journal of Mathematical Economics 10, 115 -162.

Nakamura, K.,1975. The Core of a Simple Game with Ordinal Preferences. International Journal of Game Theory 4, Issue 1/2, 95 - 104.

Nakamura, K., 1979. The vetoers in a simple game with ordinal preferences. International Journal of Game Theory 8, issue 1, 55-61.

Peleg, B., 1984. Game theoretic analysis of voting in committees. Cambridge University Press, Gambridge.

Peleg, B., 1998. Effectivity functions, game forms, games, and rights. Social Choice and Welfare 15: 67-80.

Peleg, B. and Winter, E., 2002. Constitutional implementation. Review of Economic Design 7, 187-204.

Peleg, B., 2004. Representation of Effectivity functions by acceptable game forms: a complete characterization. Mathematical Social Sciences 47, 275287.

Peleg, B. and Peters, H., 2008. Nash consistent representations of effectivity functions through lottery models. Forthcoming in Games and Economic Behavior . 